Chirurg 2012 $\cdot 83: 670$

DOI 10.1007/s00104-012-2335-5

Online publiziert: 12. Juli 2012

(c) Springer-Verlag 2012

O. Strobel · M.W. Büchler

Klinik für Allgemein-, Viszeral- und Transplantationschirurgie, Universität Heidelberg

\title{
Endoskopische transgastrische Nekrosektomie bei infizierter Pankreasnekrose
}

\section{Ist sie der chirurgischen Nekrosektomie überlegen?}

\section{Originalpublikation}

Bakker OJ, Santvoort HC van, Brunschot S van et al (2012) Endoscopic transgastric vs surgical necrosectomy for infected necrotizing pancreatitis: a randomized trial. JAMA 307:1053-1061

\section{Hintergrund und Fragestellung}

Bei Patienten mit schwerer akuter Pankreatitis und infizierter Pankreasnekrose ist eine Fokussanierung mit Entfernung des nekrotischen und infizierten Materials notwendig. Um das chirurgische Trauma und die hohen Komplikationsraten bei diesen meist kritisch kranken Patienten zu reduzieren, wird zunehmend versucht, die offen-chirurgische Nekrosektomie durch weniger invasive Verfahren wie die perkutane interventionelle Drainageneinlage und minimal-invasive laparoskopische und retroperitoneoskopische oder endoskopische Verfahren zu ersetzen oder zumindest hinauszuzögern.

In einer randomisiert-kontrollierten Studie verglichen Bakker et al. die Ergebnisse nach endoskopischer transgastrischer und chirurgischer Nekrosektomie.

\section{Methoden}

Zweiundzwanzig Patienten mit infizierter Pankreasnekrose wurden in die Gruppen „endoskopische transgastrische $\mathrm{Ne}$ krosektomie“ (ETN; n=10) und „chirurgische Nekrosektomie“ (CN; n=12) randomisiert. Im Rahmen eines „step-up approach" wurde eine Nekrosektomie bei fehlender Besserung nach perkutaner interventioneller Drainage indiziert.
In der CN-Gruppe wurden 6 Patienten retroperitoneoskopisch und 4 Patienten offen-chirurgisch nekrosektomiert; 2 in die CN-Gruppe randomisierte Patienten wurden bei Besserung nach interventioneller Drainage sekundär ausgeschlossen. Zwei Patienten in der ETN-Gruppe wurden bei fehlender Besserung nach 5 und 7 ETN-Sitzungen chirurgisch (retroperitoneoskopisch) nekrosektomiert, jedoch in der ETN-Gruppe analysiert. Der primäre Endpunkt waren die postinterventionellen Interleukin-6-Spiegel als Parameter der Entzündungsreaktion. Der kombinierte sekundäre Endpunkt war die Inzidenz von schweren Komplikationen und Tod.

\section{Ergebnisse}

Bei 95\% der Patienten bestätigte sich eine infizierte Pankreasnekrose. Die postinterventionellen Interleukin-6-Spiegel waren nach ETN signifikant niedriger. Schwere Komplikationen und Tod traten in der ETN-Gruppe signifikant weniger häufig auf als in der CN-Gruppe (20 vs. $80 \%$ ). Insbesondere wurden ein neu aufgetretenes Multiorganversagen und Pankreasfisteln in der ETN-Gruppe weniger häufig beobachtet.

\section{Fazit}

Die Autoren folgern, dass die ETN im Vergleich zur chirurgischen Nekrosektomie die Entzündungsreaktion und das Auftreten von schweren Komplikationen und Tod reduziert.
Die präsentierten Ergebnisse nach ETN sind ermutigend, müssen jedoch - wie die Autoren selbst einräumen - als präliminär eingeordnet werden. Die Studie wurde an einem sehr kleinen Patientenkollektiv durchgeführt und die Gruppen waren bezüglich wichtiger Parameter nicht vergleichbar: Der Schweremarker C-reaktives Protein war in der CNGruppe deutlich höher als in der ETNGruppe (232 vs. $141 \mathrm{mg} / \mathrm{l}$ ) und die Anzahl der Patienten mit fehlender Besserung nach vorheriger perkutaner Drainage war in der $\mathrm{CN}$-Gruppe doppelt so hoch wie in der ETN-Gruppe (8 vs. 4). Dies lässt vermuten, dass die Patienten in der CN-Gruppe schwerer und komplexer erkrankt waren. Zudem könnte die „Intention-to-treat"-Analyse der beiden letztendlich chirurgisch nekrosektomierten Patienten in der ETN-Gruppe die Gesamtergebnisse angesichts der kleinen Fallzahl verfälschen.

Die in dieser Studie gezeigten, ermutigenden Ergebnisse der endoskopischen Nekrosektomie müssen in weiteren randomisierten Studien mit höherer Fallzahl überprüft werden.

\section{Korrespondenzadresse}

\section{PD Dr. O. Strobel}

Klinik für Allgemein-,

Viszeral- und Transplantationschirurgie, Universität Heidelberg,

Im Neuenheimer Feld 110, 69120 Heidelberg

Oliver.Strobel@med.uni-heidelberg.de

Interessenkonflikt. Keine Angaben 